\title{
miR-1246 and miR-4644 in salivary exosome as potential biomarkers for pancreatobiliary tract cancer
}

\author{
TATSUYA MACHIDA ${ }^{1}$, TAKAAKI TOMOFUJI ${ }^{1,2}$, TAKAYUKI MARUYAMA $^{3}$, TOSHIKI YONEDA $^{1}$, \\ DAISUKE EKUNI ${ }^{1}$, TETSUJI AZUMA ${ }^{1}$, HISATAKA MIYAI ${ }^{1}$, HIROFUMI MIZUNO ${ }^{1}$, HIRONARI KATO ${ }^{4}$, \\ KOICHIRO TSUTSUMI ${ }^{4}$, DAISUKE UCHIDA ${ }^{4}$, AKINOBU TAKAKI ${ }^{4}$, HIROYUKI OKADA $^{4}$ and MANABU MORITA ${ }^{1}$ \\ ${ }^{1}$ Department of Preventive Dentistry, Okayama University Graduate School of Medicine, Dentistry and \\ Pharmaceutical Sciences; ${ }^{2}$ Advanced Research Center for Oral and Craniofacial Sciences, Okayama University Dental School; \\ ${ }^{3}$ Center of Innovative Clinical Medicine, Okayama University Hospital; ${ }^{4}$ Department of Gastroenterology and Hepatology, \\ Okayama University Graduate School of Medicine, Dentistry and Pharmaceutical Sciences, Okayama 700-8558, Japan
}

Received March 31, 2016; Accepted May 12, 2016

DOI: $10.3892 / o r .2016 .5021$

\begin{abstract}
Pancreatobiliary tract cancer is a highly fatal cancer. Detection of pancreatobiliary tract cancer is difficult because it lacks typical clinical symptoms and because of its anatomical location. Biomarker discovery is therefore important to detect pancreatobiliary tract cancer in its early stage. A study demonstrated that expression levels of miR-1246, miR-3976, miR-4306, and miR-4644 in serum exosomes were higher in pancreatic cancer patients than these levels in healthy control participants. Supposing that microRNA (miRNA) expression profiles in saliva are similar to those in serum, four miRNAs (miR-1246, miR-3976, miR-4306, and miR-4644) in salivary exosomes may also be useful for detection of pancreatobiliary tract cancer. In this study, it was examined whether these miRNAs could be used as biomarkers for pancreatobiliary tract cancer. Twelve pancreatobiliary tract cancer patients and 13 healthy control participants were analyzed as a cancer and a control group, respectively. Unstimulated whole saliva was collected, salivary exosomes were isolated, and total RNA was extracted. Using quantitative real-time PCR (RT-qPCR), the relative expression ratios of miR-1246 and miR-4644 were significantly higher in the cancer group than these ratios in the control group. Receiver operating characteristic (ROC)
\end{abstract}

Correspondence to: Dr Takaaki Tomofuji, Department of Preventive Dentistry, Okayama University Graduate School of Medicine, Dentistry and Pharmaceutical Sciences, 2-5-1 Shikata-cho, Kita-ku, Okayama 700-8558, Japan

E-mail: tomofu@md.okayama-u.ac.jp

Abbreviations: AUC, area under the curve; BMI, body mass index; CA19-9, carbohydrate antigen 19-9; CEA, carcinoembryonic antigen; CRP, C-reactive protein; HbAlc, hemoglobin A1c; ROC, receiver operating characteristic; $\mathrm{RT}$, reverse transcription; miRNAs, microRNAs; $\mathrm{Ct}$, threshold cycle

Key words: miR-1246, miR-4644, biomarkers, microRNAs, saliva, salivary exosomes, neoplasms, case-control studies, pancreatobiliary cancer curves were constructed to analyze the discrimination power of these miRNAs. For miR-1246, the results yielded an area under the curve (AUC) of $0.814(\mathrm{P}=0.008)$. For miR-4644, the results yielded an AUC of $0.763(\mathrm{P}=0.026)$. For the combination of miR-1246 and miR-4644, the results yielded an increased AUC of $0.833(\mathrm{P}=0.005)$. This pilot study suggests that miR-1246 and miR-4644 in salivary exosomes could be candidate biomarkers for pancreatobiliary tract cancer.

\section{Introduction}

Pancreatobiliary tract cancer is a term used to describe malignant carcinoma in pancreatic, gallbladder, and extrahepatic bile ducts. Pancreatobiliary tract cancer is a highly fatal cancer (1-4). Detection of pancreatobiliary tract cancer is difficult because it lacks typical clinical symptoms and because of its anatomical location, especially in the early stage. Biomarker discovery is therefore important for identification of increased risk, early diagnosis, and prediction of response to therapy and prognosis of pancreatobiliary tract cancer $(5,6)$. The serum carbohydrate antigen 19-9 (CA19-9) level is one clinically useful biomarker related to pancreatobiliary tract cancer (6-8). However, there is also still a limit to detect pancreatobiliary tract cancer using the existing methods (9). Therefore, further studies are needed to develop biomarkers for pancreatobiliary tract cancer.

Recently, microRNAs (miRNAs) have been reported as potential biomarkers for various types of cancers including pancreatobiliary tract cancer (10-15). They are single-stranded non-coding RNAs of $21-23$ nucleotides in length that can affect gene expression at the post-transcriptional level by binding to the three prime untranslated region (3'-UTR) of target messenger RNAs (16). It has been noted that miRNAs could be detected in body fluids (17). Several studies have suggested that miRNAs circulating in blood are useful as biomarkers for the diagnosis of pancreatic cancer (10-14,18).

Recent studies have also focused on salivary miRNAs as biomarkers for various diseases (19). Saliva collection is simple and non-invasive. Saliva contains proteins, nucleic acids, and hormones originating from both local and systemic 
sources. Notably, salivary miRNAs have been shown to be highly stable because exosomes or protein complexes protect them (20). A few studies have suggested that salivary miRNAs are useful as biomarkers for the diagnosis of pancreatic cancer $(21,22)$. However, in these studies, exosomes were not extracted from saliva. Most miRNAs in human saliva likely exist in exosomes $(23,24)$. For diagnosis of pancreatobiliary tract cancer, miRNAs in salivary exosomes may be superior to those in saliva.

To the best of our knowledge, there is no literature regarding the relationship between miRNAs in salivary exosomes and pancreatobiliary tract cancer. On the other hand, one report is available regarding the relationship between miRNAs in blood exosomes and pancreatic cancer (18). In that study, four miRNAs (miR-1246, miR-3976, miR-4306, and miR-4644) in serum exosomes were identified as biomarkers for pancreatic cancer. miRNA expression profiles in saliva could be similar to those in serum (25). Therefore, in the present study, we hypothesized that miR-1246, miR-3976, miR-4306, and miR-4644 in salivary exosomes might be useful biomarkers for pancreatobiliary tract cancer. Thus, the aim of this study was to examine whether these four miRNAs in salivary exosomes could be useful biomarkers for pancreatobiliary tract cancer.

\section{Materials and methods}

Study population. The study design was a case-control study. It was not possible to estimate the sample size preliminarily, because there was no prior information on which to base a sample size (26).

Twelve patients ( 6 males and 6 females) with pancreatobiliary tract cancers were referred to the Department of Preventive Dentistry, Okayama University Hospital for saliva collection in the morning before the onset of cancer therapy from July 2013 to July 2014.

As a control group, 13 healthy participants were recruited at the Department of Preventive Dentistry, Okayama University Hospital from August 2014 to December 2014 for saliva collection in the morning. Inclusion criteria for healthy control participants were $>50$ years of age and no history of any cancer. To avoid the effect of systemic conditions on circulating miRNA expressions, the exclusion criteria for healthy control participants were as follows: diabetes (27); pulmonary diseases (28); cardiovascular diseases (29); kidney diseases (30); liver diseases (31); and autoimmune diseases (32) at the time of saliva collection. According to the inclusion/exclusion criteria, the control participants with hypertension and dyslipidemia ( 2 participants) and hyperuricemia (1 participant) were included in this study. This study was approved by the Ethics Committee, Okayama University Graduate School of Medicine, Dentistry and Pharmaceutical Sciences and Okayama University Hospital (no. 1506-052). Written, informed consent was obtained from all participants.

General status examination. Measurements were performed before the onset of cancer therapy. Medical charts were reviewed to obtain information about cancer and the body mass index (BMI). A personal interview was performed to gather information about smoking habits (pack-years). Serum levels of hemoglobin A1c (HbA1c), C-reactive protein (CRP), albumin, carcinoembryonic antigen (CEA), and CA19-9 were evaluated. Concentrations of HbA1c, CRP, and albumin were measured by the high-performance liquid chromatography method, the latex agglutination method, and the bromocresol green albumin method, respectively. Concentrations of CEA and CA19-9 were measured by electrochemiluminescence immunoassays.

Saliva collection. Unstimulated whole saliva was collected as reported previously with minor modification (24). Briefly, saliva was collected in the morning (7:00 a.m.-12:00 noon). During the collection period, participants were seated straight up and instructed to refrain from speaking or swallowing. They allowed the saliva to accumulate in the floor of the mouth and then spit it through a funnel into a tube kept on ice. At least $0.5 \mathrm{ml}$ of unstimulated whole saliva was collected. After collection of saliva, it was stored at $4^{\circ} \mathrm{C}$ for up to $6 \mathrm{~h}$, after which it was stored at $-80^{\circ} \mathrm{C}$ until use.

Exosome isolation. Exosomes were isolated from saliva samples (0.5-1.0 $\mathrm{ml})$ using Total Exosome Isolation Reagent (Invitrogen, Carlsbad, CA, USA), in accordance with the manufacturer's instructions (33).

RNA extraction. Total Exosome RNA and Protein Isolation Kits (Invitrogen) were used for extraction of total RNA from exosome samples. Total RNA was extracted in accordance with the manufacturer's instructions. After extraction of total RNA from salivary exosomes, their quality was confirmed using the Agilent 2100 Bioanalyzer and the Agilent RNA 6000 Pico Kit (both from Agilent Technologies, Santa Clara, CA, USA).

Quantitative real-time PCR (RT- $q P C R)$. To compare miRNA expression between the control and cancer group, TaqMan RT-qPCR assays were performed. TaqMan MicroRNA Assays (Life Technologies, Carlsbad, CA, USA) were used for the RT-qPCR analyses performed on the Mx3000P Real-Time QPCR System (Agilent Technologies) according to the manufacturer's instructions $(34,35)$. Briefly, reverse transcription (RT) enzymes including specific RT primers for each miRNA and total RNA sample were mixed in a tube. RT run conditions were then set. The plate was incubated at $16^{\circ} \mathrm{C}$ for $30 \mathrm{~min}, 42^{\circ} \mathrm{C}$ for $30 \mathrm{~min}$, and $85^{\circ} \mathrm{C}$ for $5 \mathrm{~min}$, and then held at $4^{\circ} \mathrm{C}$. Products of the RT reaction were stored at $-20^{\circ} \mathrm{C}$ until use. RT-qPCR was performed in triplicate using TaqMan Universal Master Mix II, no UNG (Applied Biosystems/Life Technologies, Carlsbad, CA, USA). After holding at $95^{\circ} \mathrm{C}$ for $10 \mathrm{~min}, 50$ thermal cycles $\left(95^{\circ} \mathrm{C}\right.$ for $15 \mathrm{sec}$ and $60^{\circ} \mathrm{C}$ for $\left.60 \mathrm{sec}\right)$ were run. Threshold cycle $(\mathrm{Ct})$ values were determined using the background-based threshold (cycle-range, 5-8) calculated by the MxPro Mx3000P v4.10 software (Stratagene/Agilent Technologies, Edinburgh, UK). Data with a raw $\mathrm{Ct}>40$ for each miRNA were treated as $\mathrm{Ct}=40$ for the subsequent statistical test (36). U6 snRNA was considered suitable as an internal control (22). The relative expression rates of each miRNA were calculated using U6 snRNA expression.

Discrimination power of candidate miRNA biomarkers for pancreatobiliary tract cancer. To evaluate the discrimination power of candidate miRNA biomarkers for pancreatobiliary 
tract cancer, receiver operating characteristic (ROC) curves (37) were constructed. The ROC curves were obtained by plots of the sensitivity (true-positive rate) and 1-specificity (false-positive rate) of the diagnostic test at various cut-off values. The ROC curves graphically show the relationship between the true- and the false-positive rate according to the various cut-off values. The area under the curve (AUC) was estimated to assess the predictive power. To decide the optimal threshold value, the Youden index (sensitivity + specificity -1 ) was used (38).

Statistical analyses. Characteristics of the control and cancer group are represented as continuous variables [age, BMI, smoking habit (pack-years), HbA1c, CRP, albumin, CEA, and CA19-9] and categorical variables (gender, cancer site, and cancer stage). The Mann-Whitney U test, the Chi-square test, and Fisher's exact test were used to assess significant differences in clinical variables between the two groups, as appropriate. The Mann-Whitney $U$ test was used to compare the relative expression ratios of each miRNA of the control and cancer group. To calculate the P-value for the AUC, a non-parametric test for $\mathrm{AUC}=0.5$ was performed. Two-sided $\mathrm{P}<0.05$ values were considered to represent significant differences. To assess the correlations of variables in the cancer group, Spearman's rank correlation coefficient and its $\mathrm{P}$-value were calculated for age (years), HbAlc (NGSP) (\%), CRP (mg/dl), albumin (g/dl), CEA (ng/ml), CA19-9 (U/ml), relative expression ratios of the miRNAs, and smoking (pack-years) as continuous variables, and cancer stage (I-III, IVa, and IVb) as a categorical variable. These statistical analyses were performed using SPSS software version 20 (SPSS, Inc., Chicago, IL, USA).

\section{Results}

Characteristics of the participants. Table I summarizes the characteristics of all participants. There were no significant differences in age and gender between the two groups. However, a significant difference in the percent of current smokers was observed $(\mathrm{P}=0.015)$. Pancreatic cancer was predominant in the patient group $(75.0 \%$ ), and $83.3 \%$ of patients were diagnosed as having the most advanced (IVa or IVb) stage. In addition, 58.3 and $66.7 \%$ of patients showed values greater than the reference values of CEA (10 ng/ml) and CA19-9 (100 U/ml), respectively.

$R T$-qPCR validation. After the RT-qPCR assays, miR-3976 was excluded from the following analyses because 24 of 25 (96.0\%) samples had high (>40) raw Ct-values. The number of samples having high $(>40)$ raw Ct-values of expression levels of miR-1246, miR-4306, miR-4644, and U6 snRNA were 0, 2, 1 and 0, respectively. Therefore, miR-1246, miR-4306, and miR-4644 were considered as candidate miRNAs. For the control group, the median (25th and 75 th percentile) values of $\log 2$-transformed relative expression of miR-1246, miR-4306, and miR-4644 were 11.6 (10.9, 12.6), -5.4 (-6.8, $-3.9)$, and $-6.5(-7.6,-4.9)$, respectively. For the cancer group, those of miR-1246, miR-4306, and miR-4644 were 14.7 (12.6, 16.2), $-5.0(-8.1,-2.9)$, and $-4.1(-5.7,-2.2)$, respectively. The Mann-Whitney $U$ test showed that the relative expression ratios of miR-1246 and miR-4644 were significantly higher in the cancer than these ratios in the control group (Fig. 1).
Table I. Characteristics of the control and cancer group.

\begin{tabular}{|c|c|c|c|}
\hline Characteristics & $\begin{array}{l}\text { Control } \\
(n=13)\end{array}$ & $\begin{array}{l}\text { Cancer } \\
(n=12)\end{array}$ & $\mathrm{P}-$ value $^{\mathrm{a}}$ \\
\hline \multicolumn{4}{|l|}{ Age (years) } \\
\hline Median (range) & $66(53-83)$ & $65(45-84)$ & 0.728 \\
\hline \multicolumn{4}{|l|}{ Gender n (\%) } \\
\hline Male & $6(46.2)$ & $6(50.0)$ & 0.848 \\
\hline Female & $7(53.8)$ & $6(50.0)$ & \\
\hline \multicolumn{4}{|l|}{ Smoking, n (\%) } \\
\hline Current & $0(0.0)$ & $5(41.7)$ & 0.015 \\
\hline \multicolumn{4}{|l|}{ Cancer site, n (\%) } \\
\hline Bile duct cancer & & $2(16.7)$ & \\
\hline Gallbladder cancer & & $1(8.3)$ & \\
\hline Pancreatic cancer & & $9(75.0)$ & \\
\hline \multicolumn{4}{|l|}{$\mathrm{T}_{\text {category }}^{\mathrm{b}}, \mathrm{n}(\%)$} \\
\hline $\mathrm{T} 1$ & & $0(0.0)$ & \\
\hline $\mathrm{T} 2$ & & $1(8.3)$ & \\
\hline $\mathrm{T} 3$ & & $2(16.7)$ & \\
\hline $\mathrm{T} 4$ & & $7(58.3)$ & \\
\hline Unknown & & $2(16.7)$ & \\
\hline \multicolumn{4}{|l|}{$\mathrm{N}$ category $^{\mathrm{b}}, \mathrm{n}(\%)$} \\
\hline No & & $6(50.0)$ & \\
\hline N1 & & $1(8.3)$ & \\
\hline $\mathrm{N} 2$ & & $1(8.3)$ & \\
\hline N3 & & $1(8.3)$ & \\
\hline Unknown & & $3(25.0)$ & \\
\hline \multicolumn{4}{|l|}{ M category ${ }^{\mathrm{b}}, \mathrm{n}(\%)$} \\
\hline M0 & & $6(50.0)$ & \\
\hline M1 & & $5(41.7)$ & \\
\hline Unknown & & $1(8.3)$ & \\
\hline \multicolumn{4}{|l|}{ Cancer stage $^{\mathrm{b}}, \mathrm{n}(\%)$} \\
\hline I & & $0(0.0)$ & \\
\hline II & & $1(8.3)$ & \\
\hline III & & $1(8.3)$ & \\
\hline IVa & & $4(33.3)$ & \\
\hline $\mathrm{IVb}$ & & $6(50.0)$ & \\
\hline \multicolumn{4}{|l|}{ HbA1c (NGSP) (\%) } \\
\hline Median (range) & & $5.9(4.9-8.1)$ & \\
\hline \multicolumn{4}{|l|}{$\mathrm{CRP}(\mathrm{mg} / \mathrm{dl})$} \\
\hline Median (range) & & $0.42(0.06-5.47)$ & \\
\hline \multicolumn{4}{|l|}{ Albumin (g/dl) } \\
\hline Median (range) & & $3.7(2.9-4.5)$ & \\
\hline \multicolumn{4}{|l|}{ CEA (ng/ml) } \\
\hline Median (range) & & $12(1-1,379)$ & \\
\hline \multicolumn{4}{|l|}{ CA19-9 (U/ml) } \\
\hline Median (range) & & $414(1-38,864)$ & \\
\hline
\end{tabular}

${ }^{a}$ Mann-Whitney U test, Chi-square test, or Fisher's exact test was used. ${ }^{b}$ General Rules for the Study of Pancreatic Cancer (Japan Pancreas Society) were used. HbA1c, hemoglobin A1c; CRP, C-reactive protein; CEA, carcinoembryonic antigen; CA19-9, carbohydrate antigen 19-9. 
A
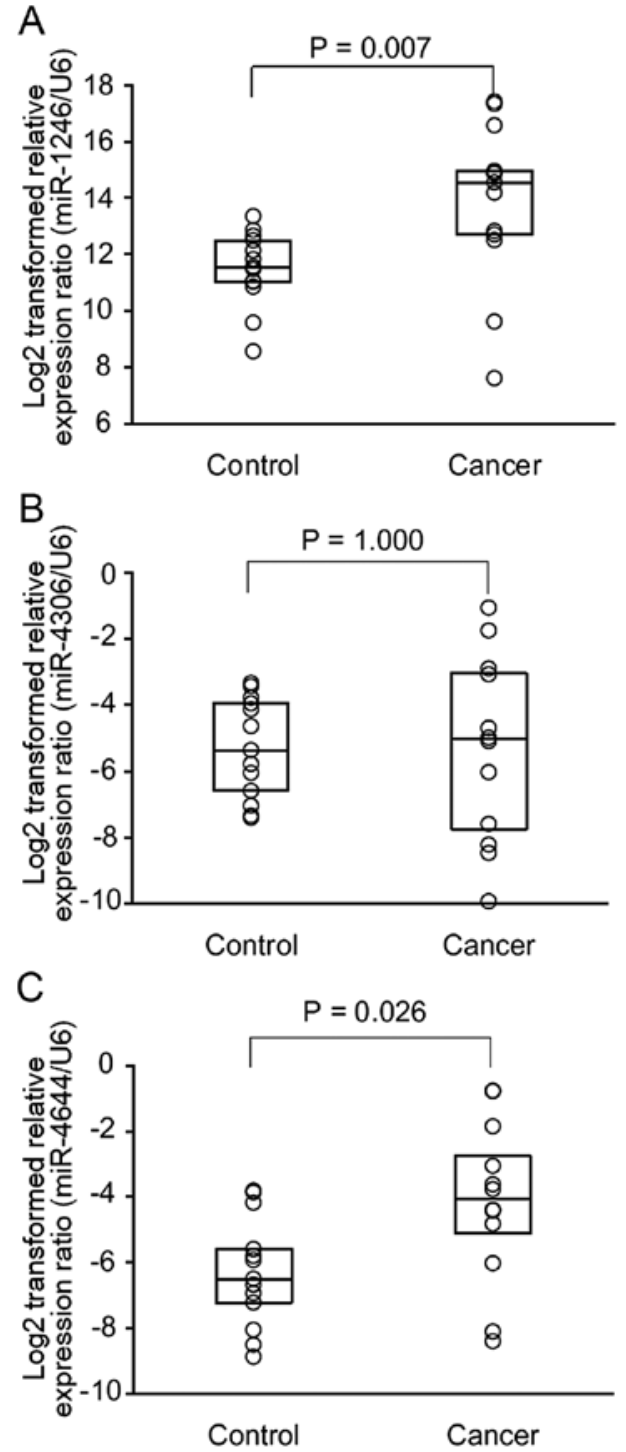

Figure 1. Differential relative expression ratio of miRNAs obtained in the control $(n=13)$ and cancer $(n=12)$ group. U6 snRNA was used as reference. Log2-transformed relative expression ratio of (A) miR-1246, (B) miR-4306 and (C) miR-4644 for each sample is represented as plots. The horizontal lines in the boxes represent the 25th, 50th and 75th percentile values of $\log 2$-transformed relative expression ratio of each miRNA, respectively. The P-value was calculated using Mann-Whitney U test. miRNAs, microRNAs.

Discrimination power of miR-1246 and miR-4644 for pancreatobiliary tract cancer. To evaluate the discrimination power of miR-1246 and miR-4644 for pancreatobiliary tract cancer, ROC curves were constructed (Fig. 2). For miR-1246, the results yielded an AUC of 0.814 [95\% confidence interval (CI), 0.616-1.000; cut-off, 13.77; sensitivity, 0.667 ; specificity, 1.000 ; $\mathrm{P}=0.008]$. For miR-4644, the results yielded an AUC of 0.763 (95\% CI, 0.564-0.961; cut-off, -5.205; sensitivity, 0.750; specificity, $0.769 ; \mathrm{P}=0.026$ ). For the combination of miR-1246 and miR-4644, the results yielded an increased AUC of 0.833 (95\% CI, 0.630-1.000; cut-off, 8.035; sensitivity, 0.833; specificity, 0.923; $\mathrm{P}=0.005)$.

Correlations of variables in the cancer group. In the cancer group, there were significant correlations between CRP and cancer stage $(r=0.822)$, between albumin and $\mathrm{HbAlc}$
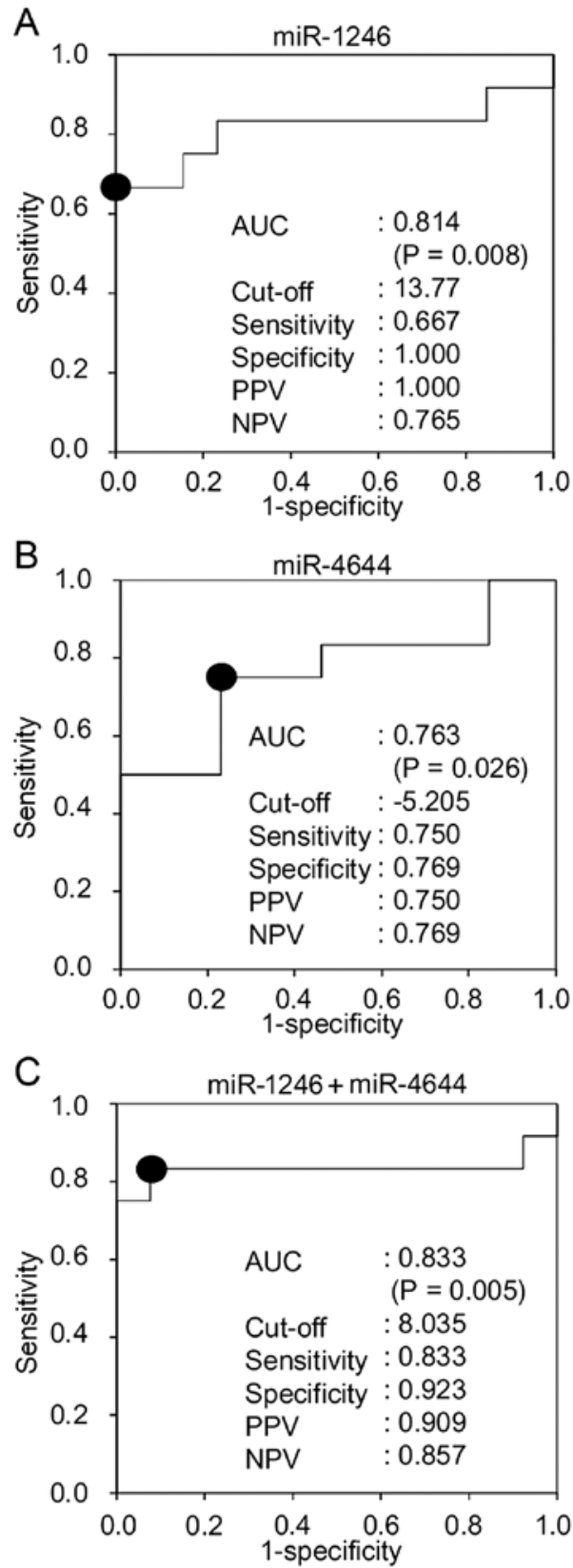

Figure 2. ROC curve analysis for the discriminatory power of salivary exosomal miRNAs for the control and cancer group. ROC curves for (A) miR-1246, (B) miR-4644 and (C) combination of miR-1246 and miR-4644 are represented. $\log 2$-transformed relative expression ratio using U6 snRNA as reference was used for each miRNA. P-value was calculated using non-parametric test for AUC $=0.5$. Cut-off, sensitivity, specificity, PPV, and NPV were optimized using the maximum Youden index. Black circle represents the plot with these optimized values. ROC, receiver operating characteristic; miRNAs, microRNAs; AUC, area under the curve; PPV, positive predictive value; NPV, negative predictive value.

$(\mathrm{r}=0.582)$, between albumin and CRP $(\mathrm{r}=-0.711)$, between CA19-9 and miR-1246 ( $r=0.818)$, between miR-1246 and miR-4644 $(r=0.671)$, between smoking and HbAlc $(r=0.579)$, and between smoking and albumin $(\mathrm{r}=0.647)$ (Table II).

\section{Discussion}

This study was performed to clarify whether miR-1246, miR-3976, miR-4306, and miR-4644 levels in salivary 


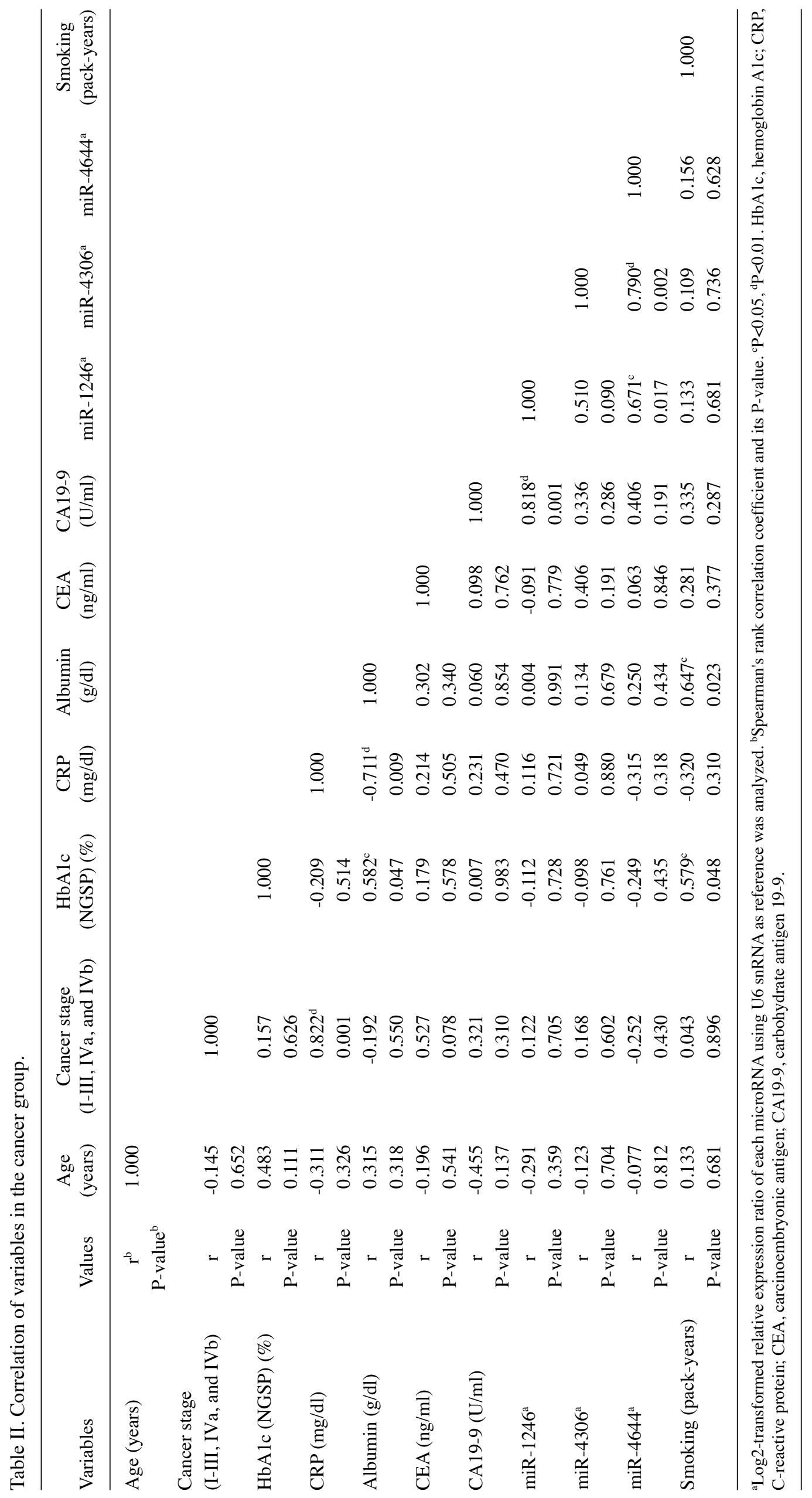


exosomes were useful as potential biomarkers for pancreatobiliary tract cancer. Among these four miRNAs, two miRNAs (miR-1246 and miR-4644) in salivary exosomes showed significantly higher expression in pancreatobiliary tract cancer patients than that noted in healthy control participants. In addition, in the ROC curve analysis, the AUCs of both miR-1246 and miR-4644 were $>0.7$, indicating fair discriminatory power (39). These results indicate that miR-1246 and miR-4644 in salivary exosomes could be useful biomarkers for screening pancreatobiliary tract cancer patients. On the other hand, in the present study, overlap of the relative expression profiles of miR-1246 and miR-4644 was also found in the cancer and control groups. This suggests that miR-1246 and miR-4644 might be non-specific screening biomarkers for pancreatobiliary tract cancer.

Compared to other body fluids including blood, saliva may be immediately exposed to the outside environment and confounded by a wide variety of environmental factors (19). However, some molecules in saliva are highly stable and represent the body's health status. In this study using salivary exosomes, the sensitivity and specificity for detecting pancreatobiliary tract cancer were 66.7 and $100.0 \%$ for miR-1246 (cut-off, 13.77) and 75.0 and $76.9 \%$ for miR-4644 (cut-off, -5.205), respectively. In serum, it is reported that sensitivity and specificity for pancreatic cancer of miR-1246 were 79.1 and $82.0 \%$, respectively (40). It is also known that, for pancreatic cancer, in plasma they were 82 and $73 \%$ for miR-885-5p, 82 and $82 \%$ for miR-22-3p, and 82 and 55\% for miR-642b-3p, respectively (11). Taking these findings into account, the sensitivity for pancreatobiliary tract cancer of miR-1246 and miR-4644 in salivary exosomes seems to be slightly lower than other miRNAs in blood, and their specificity seems to be similar or greater than that of other miRNAs in blood.

In the present findings, the combination of miR-1246 and miR-4644 in salivary exosomes increased the sensitivity for pancreatobiliary tract cancer. This is consistent with previous results, indicating that concomitant pancreatic cancer-initiating cell and miRNA marker expression strengthened the sensitivity for pancreatic cancer (18). In the present study, since drawing blood from healthy participants was difficult ethically, we could not combine miRNAs in salivary exosomes and serum markers to assess the predictive power. However, serum markers would further strengthen the sensitivity for pancreatobiliary tract cancer of miR-1246 and miR-4644 in salivary exosomes.

Serum CA19-9 is one of the most studied and validated serum biomarkers for pancreatobiliary tract cancer (6-8). In the present study, the serum CA19-9 level was significantly correlated with the expression of miR-1246 in the cancer group. This observation indicates that miR-1246 in salivary exosomes was associated with serum biomarkers for pancreatobiliary tract cancer. On the other hand, the expression of miR-4644 in salivary exosomes was significantly correlated with the expression of miR-1246, but not with serum CA19-9. The relationship between miR-4644 in salivary exosomes and the onset of pancreatobiliary tract cancer may be indirect.

miR-1246 has been found to be aberrantly expressed in pancreatic cancer tissue (41), and such a condition could contribute to the increased miRNA in blood. Due to the extensive blood supply in salivary glands, saliva is considered to be a terminal product of blood circulation, and molecules that are present in blood are also present in saliva. Therefore, it is feasible that miR-1246 in salivary exosomes originated from pancreatobiliary tract cancer tissue. However, further studies are needed to clarify this point.

Studies have investigated the relationship between salivary miRNA profiles and pancreatobiliary tract cancer. For instance, a clinical study showed that miR-21, miR-23a, miR-23b, and miR-29c were significantly upregulated in saliva of pancreatic cancer patients compared to controls (21). Another clinical study also reported that salivary miR-3679-5p was significantly downregulated and miR-940 was significantly upregulated in pancreatic cancer (22). Among the present findings, miR-1246 and miR-4644 in salivary exosomes were confirmed to show significant cancer-related increases. The present and previous findings support the notion that salivary miRNAs are useful biomarkers for pancreatobiliary tract cancer. A saliva test that is less invasive than a blood test or endoscopy may be used as a broad screening test to identify pancreatobiliary tract cancer patients who would need further screening.

The limitations of this study are as follows. First, external validity was limited because all participants were recruited at the Okayama University Hospital. In addition, the cancer patients were mainly diagnosed as having advanced-stage disease when they participated in this study. Further studies including patients with chronic pancreatitis and early stage of pancreatobiliary tract cancer are necessary to improve the validity of miR-1246 and miR-4644 in salivary exosomes as screening markers of pancreatobiliary tract cancer. Furthermore, overall screening of miRNAs in salivary exosomes using a microarray will be necessary to discover a new biomarker in pancreatobiliary tract cancer patients.

In conclusion, the present results demonstrated that miR-1246 and miR-4644 in salivary exosomes could be useful biomarkers for identification of patients with pancreatobiliary tract cancer. We hope that clinical use of simple and non-invasive salivary tests will contribute to the screening of pancreatobiliary tract cancer patients and improve their survival.

\section{Acknowledgements}

This study was supported by a Grant-in-Aid for Scientific Research (26670904) from the Ministry of Education, Culture, Sports, Science and Technology, Tokyo, Japan.

\section{References}

1. Saif MW: Advancements in the management of pancreatic cancer: 2013. JOP 14: 112-118, 2013.

2. Benavides M, Antón A, Gallego J, Gómez MA, Jiménez-Gordo A, La Casta A, Laquente B, Macarulla T, Rodríguez-Mowbray JR and Maurel J: Biliary tract cancers: SEOM clinical guidelines. Clin Transl Oncol 17: 982-987, 2015.

3. Shaffer EA: Gallbladder cancer: The basics. Gastroenterol Hepatol (N Y) 4: 737-741, 2008.

4. Im JH, Seong J, Lee IJ, Park JS, Yoon DS, Kim KS, Lee WJ and Park KR: Surgery alone versus surgery followed by chemotherapy and radiotherapy in resected extrahepatic bile duct cancer: Treatment outcome analysis of 336 patients. Cancer Res Treat 48: 583-595, 2016.

5. Poruk KE, Firpo MA, Adler DG and Mulvihill SJ: Screening for pancreatic cancer: Why, how, and who? Ann Surg 257: 17-26, 2013. 
6. Zeng $\mathrm{X}$ and Tao H: Diagnostic and prognostic serum marker of cholangiocarcinoma (Review). Oncol Lett 9: 3-8, 2015.

7. Ballehaninna UK and Chamberlain RS: The clinical utility of serum CA 19-9 in the diagnosis, prognosis and management of pancreatic adenocarcinoma: An evidence based appraisal. J Gastrointest Oncol 3: 105-119, 2012.

8. Lin MS, Huang JX and Yu H: Elevated serum level of carbohydrate antigen 19-9 in benign biliary stricture diseases can reduce its value as a tumor marker. Int J Clin Exp Med 7: 744-750, 2014.

9. Fong ZV and Winter JM: Biomarkers in pancreatic cancer: Diagnostic, prognostic, and predictive. Cancer J 18: 530-538, 2012 .

10. Ali S, Almhanna K, Chen W, Philip PA and Sarkar FH: Differentially expressed miRNAs in the plasma may provide a molecular signature for aggressive pancreatic cancer. Am J Transl Res 3: 28-47, 2010.

11. Ganepola GA, Rutledge JR, Suman P, Yiengpruksawan A and Chang DH: Novel blood-based microRNA biomarker panel for early diagnosis of pancreatic cancer. World J Gastrointest Oncol 6: 22-33, 2014.

12. Li A, Yu J, Kim H, Wolfgang CL, Canto MI, Hruban RH and Goggins M: MicroRNA array analysis finds elevated serum miR-1290 accurately distinguishes patients with low-stage pancreatic cancer from healthy and disease controls. Clin Cancer Res 19: 3600-3610, 2013.

13. Liu J, Gao J, Du Y, Li Z, Ren Y, Gu J, Wang X, Gong Y, Wang W and Kong X: Combination of plasma microRNAs with serum CA19-9 for early detection of pancreatic cancer. Int J Cancer 131: 683-691, 2012.

14. Liu R, Chen X, Du Y, Yao W, Shen L, Wang C, Hu Z, Zhuang R, Ning G, Zhang C, et al: Serum microRNA expression profile as a biomarker in the diagnosis and prognosis of pancreatic cancer. Clin Chem 58: 610-618, 2012.

15. Wang M, Wen TF, He LH, Li C, Zhu WJ and Trishul NM: A six-microRNA set as prognostic indicators for bile duct cancer. Int J Clin Exp Med 8: 17261-17270, 2015.

16. Stark A, Bushati N, Jan CH, Kheradpour P, Hodges E, Brennecke J, Bartel DP, Cohen SM and Kellis M: A single Hox locus in Drosophila produces functional microRNAs from opposite DNA strands. Genes Dev 22: 8-13, 2008.

17. Weber JA, Baxter DH, Zhang S, Huang DY, Huang KH, Lee MJ, Galas DJ and Wang K: The microRNA spectrum in 12 body fluids. Clin Chem 56: 1733-1741, 2010

18. Madhavan B, Yue S, Galli U, Rana S, Gross W, Müller M, Giese NA, Kalthoff H, Becker T, Büchler MW, et al: Combined evaluation of a panel of protein and miRNA serum-exosome biomarkers for pancreatic cancer diagnosis increases sensitivity and specificity. Int J Cancer 136: 2616-2627, 2015

19. Lin X, Lo HC, Wong DT and Xiao X: Noncoding RNAs in human saliva as potential disease biomarkers. Front Genet 6: 175, 2015.

20. Palanisamy V, Sharma S, Deshpande A, Zhou H, Gimzewski J and Wong DT: Nanostructural and transcriptomic analyses of human saliva derived exosomes. PLoS One 5: e8577, 2010

21. Humeau M, Vignolle-Vidoni A, Sicard F, Martins F, Bournet B, Buscail L, Torrisani J and Cordelier P: Salivary microRNA in pancreatic cancer patients. PLoS One 10: e0130996, 2015.

22. Xie Z, Yin X, Gong B, Nie W, Wu B, Zhang X, Huang J, Zhang $\mathrm{P}$, Zhou $\mathrm{Z}$ and Li Z: Salivary microRNAs show potential as a noninvasive biomarker for detecting resectable pancreatic cancer. Cancer Prev Res (Phila) 8: 165-173, 2015.
23. Michael A, Bajracharya SD, Yuen PS, Zhou H, Star RA, Illei GG and Alevizos I: Exosomes from human saliva as a source of microRNA biomarkers. Oral Dis 16: 34-38, 2010.

24. Gallo A and Alevizos I: Isolation of circulating microRNA in saliva. Methods Mol Biol 1024: 183-190, 2013.

25. Bahn JH, Zhang Q, Li F, Chan TM, Lin X, Kim Y, Wong DT and Xiao X: The landscape of microRNA, Piwi-interacting RNA, and circular RNA in human saliva. Clin Chem 61: 221-230, 2015.

26. Julious SA: Sample size of 12 per group rule of thumb for a pilot study. Pharm Stat 4: 287-291, 2005.

27. Chien HY, Lee TP, Chen CY, Chiu YH, Lin YC, Lee LS and Li WC: Circulating microRNA as a diagnostic marker in populations with type 2 diabetes mellitus and diabetic complications. J Chin Med Assoc 78: 204-211, 2015.

28. Sessa R and Hata A: Role of microRNAs in lung development and pulmonary diseases. Pulm Circ 3: 315-328, 2013.

29. Schulte C and Zeller T: microRNA-based diagnostics and therapy in cardiovascular disease-Summing up the facts. Cardiovasc Diagn Ther 5: 17-36, 2015.

30. Wei Q, Mi QS and Dong Z: The regulation and function of microRNAs in kidney diseases. IUBMB Life 65: 602-614, 2013.

31. Arrese M, Eguchi A and Feldstein AE: Circulating microRNAs: Emerging biomarkers of liver disease. Semin Liver Dis 35: 43-54, 2015.

32. $\mathrm{Qu} \mathrm{Z}, \mathrm{Li} \mathrm{W}$ and $\mathrm{Fu} \mathrm{B}$ : MicroRNAs in autoimmune diseases. BioMed Res Int 2014: 527895, 2014

33. Machida T, Tomofuji T, Ekuni D, Maruyama T, Yoneda T, Kawabata Y, Mizuno H, Miyai H, Kunitomo M and Morita M: MicroRNAs in salivary exosome as potential biomarkers of aging. Int J Mol Sci 16: 21294-21309, 2015.

34. Schmittgen TD, Lee EJ, Jiang J, Sarkar A, Yang L, Elton TS and Chen C: Real-time PCR quantification of precursor and mature microRNA. Methods 44: 31-38, 2008.

35. Chen C, Tan R, Wong L, Fekete R and Halsey J: Quantitation of microRNAs by real-time RT-qPCR. Methods Mol Biol 687: 113-134, 2011.

36. Life Technologies: DataAssist v3.0 Software user instructions, 2011: http://cgs.hku.hk/portal/files/CGS/Genomics/Realtime-PCR /dataassist\%20v3_0\%20software\%20user\%20instructions.pdf.

37. Hajian-Tilaki K: Receiver Operating Characteristic (ROC) Curve Analysis for medical diagnostic test evaluation. Caspian J Intern Med 4: 627-635, 2013.

38. Ruopp MD, Perkins NJ, Whitcomb BW and Schisterman EF: Youden Index and optimal cut-point estimated from observations affected by a lower limit of detection. Biom J 50: 419-430, 2008

39. Swets JA: Measuring the accuracy of diagnostic systems. Science 240: 1285-1293, 1988.

40. Kojima M, Sudo H, Kawauchi J, Takizawa S, Kondou S, Nobumasa $\mathrm{H}$ and Ochiai A: MicroRNA markers for the diagnosis of pancreatic and biliary-tract cancers. PLoS One 10: e0118220, 2015.

41. Ali S, Saleh H, Sethi S, Sarkar FH and Philip PA: MicroRNA profiling of diagnostic needle aspirates from patients with pancreatic cancer. Br J Cancer 107: 1354-1360, 2012. 